\title{
Aldosterone resistance; a rare differential diagnosis for persistent hyperkalemia
}

\begin{abstract}
In the human body, Aldosterone is an important hormone for sodium conservation in the kidneys, salivary glands, sweat glands and colon. Aldosterone is synthesized exclusively in the zona glomerulosa of the adrenal gland. Significance of aldosterone production along with its appropriate action on the receptors is undeniable as far as hemostasis of intracellular and extracellular electrolytes is concerned. Destruction or dysfunction of the adrenal gland in conditions such as primary adrenal insufficiency, congenital adrenal hypoplasia, isolated mineralocorticoid deficiency, acquired secondary aldosterone deficiency (hyporeninemic hypoaldosteronism), acquired primary aldosterone deficiency and inherited enzymatic defects in aldosterone biosynthesis cause clinical symptoms and laboratory characteristics owing to aldosterone deficiency. Pseudohypoaldosteronism is an aldosterone resistance syndrome i.e. a condition due to insensitivity of target tissues to aldosterone resulting in hyponatremia, hyperkalemia and metabolic acidosis. For a proper diagnosis of Aldosterone resistance; serum Sodium levels, serum Potassium levels, ACTH levels, plasma Renin and Aldosterone activity along with serum Cortisol levels play key roles. High doses of Fludrocortisone therapy helps in overcoming aldosterone resistance and assist in maintaining electrolyte balance in the body.
\end{abstract}

Keywords: hypoaldosteronism, hyponatremia, hyperkalemia, aldosterone resistance, pseudohypoaldosteronism
Volume 5 Issue 5 - 2017

\section{Muhammad Umair, Afsoon Razavi, Zehra Tekin, Issac Sachmechi \\ Department of Medicine, Icahn School of Medicine, USA}

Correspondence: Muhammad Umair, Department of Medicine, Icahn School of Medicine at Mount Sinai/NYC Health + Hospital/Queens Jamaica, Diabetes center, 4th floor, Suit P-432, pavilion building, Queens hospital center, 82-68 164th street, Jamaica, New York, USA, Tel + I5 I63436454, Email muhammadumair00I7@gmail.com

Received: July 25, 2017 | Published: August 09, 2017
Abbreviations: Na-K ATPase Pump, sodium potassium ATPase pump; ACTH, adrenocorticotropic hormone; WNK Kinase protein, with no $(\mathrm{K})$ lysine kinase protein [lysine deficient protein kinase]

\section{Introduction}

Aldosterone is an important hormone for sodium conservation in the kidney, salivary glands, sweat glands and colon. In normal states within the kidney, aldosterone increases sodium reabsorption through opening epithelial sodium channels within the principle cells of the cortical collecting tubule causing the development of an electronegative potential in the lumen. This results in an electrical gradient that favors secretion of cellular potassium through the potassium channels. It also promotes active sodium transport and excretion of potassium via the Mineralocorticoid receptor (MR) and results in activation of the Na-K ATPase pump in the target tissues. In hypoaldosterone states, there is reduced sodium reabsorption in the cortical collecting tubules; therefore a lesser electrical gradient forms to facilitate secretion of cellular potassium, which results in hyperkalemia along with hyponatremia and acidosis. ${ }^{1}$ Many patients with hyperkalemia have a readily identifiable cause, not limited to acute and chronic kidney disease but also include adrenal insufficiency, metabolic acidosis, insulin deficiency, rhabdomyolysis, burn injuries, intravascular hemolysis and medications, which leads to appropriate management. In others, particularly those having a reduced glomerular filtration rate, differentiating between (relative) hypoaldosteronism (HA) and renal aldosterone resistance (RAR) can be problematic. ${ }^{1-3}$

\section{Case presentation}

A 63years old male was first seen in the clinic with alleged diagnosis of adrenal insufficiency. He was started on fludrocortisone (0.1mg q daily) and hydrocortisone (25mg q AM and $12.5 \mathrm{mg} \mathrm{q} P M)$ by an outside physician but self-discontinued Hydrocortisone a few months prior to his visit. Patient was not on any antibiotics or diuretic treatment and was found to have low-normal blood pressure, normoglycemia, hyperkalemia, hyponatremia, and metabolic acidosis. Further testing revealed decreased early morning cortisol level of $11.7 \mathrm{mcg} / \mathrm{dL}$ ( 0.7 to $22.4 \mathrm{mcg} / \mathrm{dL}$ ) and ACTH level of $20 \mathrm{pg} / \mathrm{ml}$ $(0-46 \mathrm{pg} / \mathrm{mL})$. ACTH stimulation test was done and baseline cortisol level was $10.89 \mathrm{mcg} / \mathrm{dL}$, followed by $15.47 \mathrm{mcg} / \mathrm{dL}$ at 30 minutes and $19.72 \mathrm{mcg} / \mathrm{dL}$ at 60 minutes as well as aldosterone level was $111.7 \mathrm{ng} /$ $\mathrm{dL}$ at 30 minutes and $249.2 \mathrm{ng} / \mathrm{dL}$ at 60 minutes $(0-30 \mathrm{ng} / \mathrm{dL})$ after stimulation test. Additional tests revealed negative 21-Hydroxylase antibodies and elevated plasma renin activity of $78 \mathrm{ng} / \mathrm{ml} / \mathrm{hr}(0.2-$ $3.3 \mathrm{ng} / \mathrm{ml} / \mathrm{hr}$ ). Patient was continued on fludrocortisone. He presented to clinic in following visit after missing his medication for 2 weeks and found to have low blood pressure $(80 / 58 \mathrm{mmHg})$, hyperkalemia and metabolic acidosis. Resuming his fludrocortisone normalized his blood pressure.

\section{Discussion}

Clinical presentation of hypoaldosteronism can manifest either with aldosterone deficiency or due to aldosterone resistance. Aldosterone deficiency results in conditions like hyporeninemic hypoaldosteronism (due to diabetic kidney disease, non-steroidal anti-inflammatory drugs, calcineurin inhibitors), angiotensin inhibitors, heparin therapy, primary adrenal insufficiency, critical illness, congenital isolated hypoaldosteronism, and pseudohypoaldosteronism type-2 (Gordon's syndrome or familial hyperkalemic hypertension) that is due to abnormalities in WNK kinases in the distal nephrons which increase chloride reabsorption leading to reduced renal potassium secretion and is characterized by hypertension, hyperkalemia, metabolic acidosis, normal renal function, and low or low-normal plasma renin 
activity and aldosterone concentrations. ${ }^{1,2,4}$ Whereas, aldosterone resistance occurs due to inhibition of the epithelial sodium channel (potassium-sparing diuretics, trimethoprim, pentamidine) and pseudohypoaldosteronism type- 1 that is characterized by marked elevations of plasma aldosterone levels..$^{1,2,5-8}$ Hypoaldosteronism can be due to an autosomal recessive inheritance pattern, an autosomal dominant pattern or via sporadic form. The autosomal dominant form tends to be associated with milder symptoms. ${ }^{1,2}$ (Table 1).

Table I Diagnostic findings for major causes of hypoaldosteronism.'

\begin{tabular}{|c|c|c|}
\hline Disorder & $\begin{array}{l}\text { Plasma renin } \\
\text { activity }\end{array}$ & Plasma aldosterone \\
\hline $\begin{array}{l}\text { Hyporeninemic } \\
\text { hypoaldosteronism }\end{array}$ & Low to normal & Low \\
\hline Primary adrenal insufficiency & High & Low \\
\hline $\begin{array}{l}\text { Pseudohypoaldosteronism } \\
\text { type } 1\end{array}$ & High & High \\
\hline $\begin{array}{l}\text { Pseudohypoaldosteronism } \\
\text { type } 2\end{array}$ & Low to normal & Low to normal \\
\hline
\end{tabular}

This case represents a challenging differential diagnosis between primary adrenal insufficiency and aldosterone resistance syndrome. Although persistent hyperkalemia and hyponatremia are more commonly seen with adrenal insufficiency but normal basal cortisol and $\mathrm{ACTH}$ levels and negative ACTH stimulation test ruled out hypoadrenalism. In our case, aldosterone levels found significantly elevated in the face of hyperkalemia and raised suspicion of aldosterone resistance syndrome. ${ }^{6}$ Fludrocortisone therapy has been approved by FDA for primary replacement in adrenocortical insufficiency (Addison's Disease), salt-losing forms of congenital adrenogenital syndrome and hyporeninemic hypoaldosteronism. It is also used off label for the treatment of severe orthostatic hypotension refractory to high salt diet and adequate fluid intake since it has vasoconstrictive effects through V1A vascular smooth muscle receptors. Patients with aldosterone resistance may also need fludrocortisone to maintain their blood pressure along with high salt diet, because high doses of fludrocortisone overcome aldosterone resistance, as it was seen in our case.

\section{Conclusion}

While hyperkalemia can be due to adrenal insufficiency, clinician should be aware of the possibility of aldosterone resistance if there is no evidence of hypocortisolemia and should not give glucocorticoids in addition to mineralocorticoids.

\section{Acknowledgements}

None.

\section{Conflict of interest}

The author declares no conflict of interest.

\section{References}

1. Rose BD, Post TW. Clinical Physiology of Acid-Base and Electrolyte Disorders. 5th ed. USA: McGraw-Hill; 2011. p. 1-992.

2. Jain G, Ong S, Warnock DG. Genetic disorders of potassium homeostasis. Semin Nephrol. 2013;33(3):300-309.

3. Willian RA. Hypothesis A simple algorithm to distinguish between hypoaldosteronism and renal aldosterone resistance in patients with persistent hyperkalemia. Nephrology. 2008;13(6):459-464.

4. DeFronzo RA. Hyperkalemia and hyporeninemic hypoaldosteronism. Kidney Int. 1980;17(1):118-134.

5. Young WF, Sterns RH, Lacroix A, et al. Etiology, diagnosis, and treatment of hypoaldosteronism (type 4 RTA). Netherlands: Wolters Kluwer; 2017.

6. Arai K, Chrousos GP. Aldosterone deficiency and resistance; 2016.

7. Throckmorton DC, Bia MJ. Pseudohypoaldosteronism: case report and discussion of the syndrome. Yale J Biol. 1991;64(3):247-254.

8. Soriano RJ, Vallo A, Oliveros R, et al. Transient pseudohypoaldosteronism secondary to obstructive uropathy in infancy. J Pediat. 1983; 103(3):375380 .

9. Bogdanovic R, Stajić N, Putnic J, et al. Transient type 1 pseudohypoaldosteronism: report on a eight-patinet series and literature review. Pediator Nephrol. 2009;24(11):2167-2175. 